\title{
Examining the association between late-life depressive symptoms, cognitive function, and brain volumes in the context of cognitive reserve
}

\author{
Deirdre M. O’Shea, Robert A. Fieo, Jamie L. Hamilton, Laura B. Zahodne, Jennifer J. Manly and Yaakov Stern \\ Cognitive Neuroscience Division, Department of Neurology and Taub Institute for Research on Alzheimer's Disease and the Aging Brain, \\ Columbia University, New York, NY, USA \\ Correspondence to: Y. Stern, E-mail: ys11@cumc.columbia.edu
}

\begin{abstract}
Objective: The present study aimed to investigate whether cognitive reserve moderated the association between depressive symptoms and cognition, as well as brain volumes in a sample of older adults.

Methods: Non-demented participants $(n=3484)$ were selected from the Washington Heights/Hamilton Heights Inwood Columbia Aging Project (Northern Manhattan). A subsample of these participants without dementia $(n=703)$, who had brain imaging data, was also selected for a separate analysis. Depressive symptomatology was assessed with the 10-item Center for Epidemiologic Studies Depression Scale. Reading level and years of education were used as measures of cognitive reserve. Four distinct cognitive composite scores were calculated: executive function, memory, visual-spatial, and language.

Results: Multiple regression analysis revealed interaction effects between both measures of cognitive reserve and depressive symptoms on all the cognitive outcome measures except for visual-spatial ability. Those with greater reserve showed greater cognitive decrements than those with lower levels of reserve as depressive symptoms increased. A borderline interaction effect was revealed between reading level and depressive symptoms on total brain volumes. Those with lower reading scores showed greater volume loss as depressive symptoms increased than those with higher reading scores.

Conclusions: Our findings indicate that the association between late-life depressive symptoms and core aspects of cognition varies depending on one's level of cognitive reserve. Those that had greater levels of education and/or reading ability showed a greater decrease in memory, executive, and language performances as depressive symptoms increased than those with lower years of education and reading ability. Copyright (C) 2014 John Wiley \& Sons, Ltd.
\end{abstract}

Key words: late life depressive symptoms; cognitive performance; cognitive reserve; brain volumes

History: Received 22 January 2014; Accepted 25 July 2014; Published online 22 August 2014 in Wiley Online Library (wileyonlinelibrary.com)

DOI: 10.1002 /gps.4192

\section{Introduction}

Individuals with late-life depression (LLD), including depressive symptoms, frequently present with neuropsychological impairment (Boone et al., 1995; Dufouil et al., 1996; Butters et al., 2004) but not always (Bieliauskas et al., 1991; Lockwood et al., 2000). Those that do present with neuropsychological impairment are particularly impaired on tasks of executive function and processing speed (Raskin, 1986; Geffen et al., 1993; Sheline et al., 2006). However, memory (Biringer et al., 2005) and visual-spatial ability (Hart et al., 1987) have also been found to be impaired in other studies. Furthermore, some investigations suggest that LLD increases the risk for continued cognitive decline and subsequent dementia, even after symptoms remit (Green et al., 2003; Ownby et al., 2006; Lee et al., 2007), while in other studies, these associations were not found (Lindsay et al., 2002; Ganguli et al., 2006; Becker et al., 2009). The question as to why depression in late life can have a pervasive negative influence on cognition in some individuals but not all remains to be reconciled. Methodological factors, including how depression is operationalized and ultimately measured, may account 
for some of these inconsistent findings. However, it is also possible that there are some moderating psychosocial factors that may influence the cognitive profile of individuals with LLD.

Mounting epidemiologic studies report factors that may protect against the onset of LLD. Factors such as educational attainment (Kessler et al., 1995; Kim et al., 2002; Ladin, 2008), socio-economic status (Turner and Lloyd, 1999; Pinquart and Sörensen, 2000), and birth weight (Thompson et al., 2001) have been shown to influence the risk of LLD. However, very few studies examined how the association between depression and cognitive performance may be moderated by these factors. The cognitive reserve (CR) hypothesis outlined by Stern (2003) provides a framework for elucidating the relationship between depression and cognitive ability in older adults. According to this hypothesis, both familial/genetic factors, such as IQ, and environmental factors, such as education, lifetime occupation, and literacy, contribute uniquely to CR. The higher an individual's CR, the better they are in coping cognitively with pathology due to disease, such as dementia. Given that there is now a growing body of literature that has linked LLD with increased white matter hyperintensity (WMH) volume (Jansson et al., 2003; for a review, see Herrmann et al., 2008) and hippocampal atrophy (Lloyd et al., 2004), as well as a decrease in total brain volume (Geerlings et al., 2012), we may also consider LLD to be a type of age-specific disease with possible distinct neurobiological substrates. Thus, the CR hypothesis may be particularly useful for explaining the variable relationship between depressive symptoms in late life and cognitive function reported in the literature.

Wight and colleagues (Wight et al., 2002) investigated how educational attainment, post-educational training, and occupation influenced cognitive function in a sample of community-dwelling older men. The authors reported that greater educational attainment moderated the influence of depressive symptoms on cognition but only when depressive symptoms were more severe. Conversely, Bhalla et al. (2005) found that educational attainment did not have a moderating effect on neuropsychological performance in a sample of depressed patients. However, the authors highlighted several methodological issues that may have influenced their results, including lacking a truly low-educated comparison group, a relatively small sample size, and a non-comprehensive neuropsychological assessment (e.g., a single test measure was used for each cognitive domain). Moreover, the authors in this study, as well as the previous studies, only included a single CR proxy in their analysis. It is possible that some measures of $\mathrm{CR}$ are more sensitive than others in different population samples. This possibility is supported by a study that demonstrated that reading level was a more sensitive indicator of CR than years of education in a ethnically diverse sample (Manly et al., 2005). These findings highlight the practical importance of using multiple measures of CR to gain a fuller understanding of its association with cognition.

Overall, there is a paucity of published research that has specifically investigated the association between $\mathrm{CR}$ and depressive symptoms on a range of cognitive performances in late life. In the present study, we sought to investigate some of these potential associations and bring some clarity to the complex relationship between LLD and cognition. In a sample of non-demented older adults, we expected that those with higher CR would be buffered against the potentially adverse influence of depressive symptoms on cognition and that this effect would be less for those with lower CR. Furthermore, a previous study, based on the same sample of participants reported in the present study, found that depressive symptoms were significantly associated with smaller overall total brain and hippocampal volumes, as well as larger $\mathrm{WMH}$ volumes (Geerlings et al., 2012). Thus, we explored whether CR would also moderate this association. We expected that greater levels of CR would attenuate the possible influence of depressive symptoms on these structural characteristics of the brain.

\section{Methods}

\section{Participants}

Participants for the present study were selected from the Washington Heights/Hamilton Heights Inwood Columbia Aging Project (WHICAP), a prospective study of aging and dementia in Medicare-eligible northern Manhattan residents. Further details on the study and sampling procedures have been described elsewhere (Tang et al., 2001). In brief, participants come from a racially and ethnically diverse population (i.e., Hispanic, African American, and non-Hispanic White) aged 65 years and older. Participants have been assessed at approximately 18-24-month intervals since the time at which the study began in 1992 (first cohort) and 1999 (second cohort).

\section{Baseline assessments}

The WHICAP participants are assessed and interviewed in person in their preferred language (i.e., English or Spanish) at each clinical visit. Each assessment is identical procedurally from which data on current 
health status, functional ability, medical history, and neurological status are obtained. Participants also received a neuropsychological test battery (Stern et al., 1992). We selected data from the baseline assessments of each potential participant for our first part of our analysis $(n=3624)$. To examine whether CR moderated the association of depressive symptoms on brain volumes, we also selected the subsample of participants $(n=769)$ who also received high resolution structural magnetic resonance imaging (MRI) for this part of our analyses (for details of this imaging project, please refer to Brickman et al., 2008).

\section{Exclusion/inclusion criteria}

We excluded individuals who had a diagnosis of dementia from both parts of our analysis (See supporting material for basis of diagnosis). In addition, data from participants with any other major neurological disorders (e.g., brain tumor, epilepsy, Parkinson's disease, Korsakoffs syndrome, and stroke) were excluded from further analysis. The final baseline sample included 3484 participants and 703 participants with MRI data. Demographic characteristics for both of these samples are presented in Table 1.

\section{Neuropsychological assessment}

Each participant received a neuropsychological battery of tests in which core cognitive abilities were assessed including memory, visual-spatial, executive function, and language. The tests included in the battery comprised subtests from widely used and standardized neuropsychological tests (for further details on how the battery was developed, please refer to Stern et al., 1992). Individual subtests for each of the four composites are described in the supplemental section.

\section{Depressive symptoms assessment}

A 10-item version of the Center For Epidemiologic Studies Depression Scale (CES-D) (Irwin et al., 1999) was used to assess depressive symptoms. Participants responded either 'yes' or 'no' to each of the questions on this scale.

\section{Cognitive reserve measures}

We used two commonly used proxies of CR: (i) level of education and (ii) reading level. Level of education was expressed as the number of years an individual reported in full time education. Reading level was
Table 1 Characteristics of the WHICAP study at baseline and at time of MRI scan

\begin{tabular}{lcc}
\hline & Baseline & MRI subsample \\
\cline { 2 - 3 } & $n=3484$ & $n=703$ \\
\cline { 2 - 3 } & Mean (SD) & Mean (SD) \\
\hline Age & $76.07(6.4)$ & $80(5.50)$ \\
Gender & & \\
Male $(n / \%)$ & $1129 / 32 \%$ & $232 / 33 \%$ \\
Female $(n / \%)$ & $2355 / 68 \%$ & $471 / 67 \%$ \\
Race & & \\
White $(n / \%)$ & $939 / 27 \%$ & $200 / 28 \%$ \\
Hispanic $(n / \%)$ & $1374 / 39 \%$ & $248 / 35 \%$ \\
African American $(n / \%)$ & $1130 / 32 \%$ & $240 / 34 \%$ \\
Other $(n / \%)$ & $41 / 1 \%$ & $15 / 2 \%$ \\
Years of education & $9.7(1.8)$ & $10.7(4.7)$ \\
& Range $=$ & Range $=$ \\
Reading level (standardized) & $0-20$ years & $0-20$ years \\
CES-D score & Median $=$ & Median $=$ \\
& $0.295(19.82)$ & $0.189(1)$ \\
BV (\% relative to ICV) & $1.8(2.01)$ & $1.3(1.7)$ \\
WMH & Range $=0-9$ & Range $=0-9$ \\
HV & - & $73 \%(4.4)$ \\
& - & $2.2(0.8)$ \\
\hline
\end{tabular}

Note: There are some missing values for some variables, so they do not always add up to $n=3484$ (baseline) or $n=703$ (MRI subsample). CES-D, Center for Epidemiologic Studies Depression Scale; BV, total brain volume; WMH, total white matter hyperintensity volume; $\mathrm{HV}$, total hippocampal volume; ICV, total intracranial volume; WHICAP, Washington Heights/Hamilton Heights Inwood Columbia Aging Project; MRI, magnetic resonance imaging.

determined for English speakers using the subtest from the Wide Range Achievement Test, $3^{\text {rd }}$ edition (Wilkinson, 1993) and the Word Accentuation Test (Del Ser et al., 1997) for Spanish speakers. Reading level scores were $z$-scored for both the English and Spanish versions and placed on the same scale.

\section{Magnetic resonance imaging procedure}

Magnetic resonance imaging was performed on participants using a $1.5 \mathrm{~T}$ Philips Intera scanner at Columbia University Medical Center. The scans were then electronically transferred to the Imaging of Dementia and Aging Laboratory at the University of California at Davis where they were processed. Measure of total brain, cranial, and $\mathrm{WMH}$ volumes were obtained from fluid attenuated inverse recovery $\mathrm{T} 2$-weighted images [repetition time $(\mathrm{TR})=11,000 \mathrm{~ms}$, echo time $(\mathrm{TE})=144.0 \mathrm{~ms}, 2800$ inversion time, field of view (FOV) $25 \mathrm{~cm}, 2$ nex, $256 \times 192$ matrix with $3 \mathrm{~mm}$ slice thickness] using procedures previously described elsewhere (DeCarli et al., 1992, 1995, 1996; Brickman 
et al., 2008). WMH volumes were log transformed to normalize their distribution. T1-weighted images acquired in the axial plane and re-sectioned coronally were used to quantify hippocampal volumes $(\mathrm{TR}=20$ $\mathrm{ms}, \mathrm{TE}=2.1 \mathrm{~ms}$, flip angle $=20 ; \mathrm{FOV} 240 \mathrm{~cm}$, $256 \times 160$ matrix with $1.3 \mathrm{~mm}$ slice thickness, no gap, voxel size $0.94 \times 1.17 \mathrm{~mm}$ in plane). For more details on this procedure and methods of analysis, refer to the supplemental section.

\section{Statistical analysis}

First, we performed separate three-step hierarchical multiple regression analyses in the baseline sample to assess whether there was an interaction between CES-D sum scores and either one of the two CR measures to predict performances on the four cognitive domains. Demographic covariates, including age, gender, and race, were included in the first step, and CES-D scores and one of the $\mathrm{CR}$ variables (i.e., education/reading) in the second step. The interaction term, CES-D by one of the CR variables, was included in the final third step. Continuous variables, that is, age, years of education, and CES-D scores, were centered by subtracting the sample mean, prior to forming the interaction term.

All statistical procedures were then repeated in the subsample of participants who had undergone MRI scans. In the first block, we controlled for age, gender, and race. CES-D scores and one of the CR variables (i.e., education and reading) were included in the second block, and the interaction term in the third block. Separate multiple regression analyses were then run on each of the four cognitive domains. However, the primary purpose of using this sample was to examine whether the CR variables moderated the potential association of depressive symptoms on the brain. Therefore, following the analyses and using the cognitive outcome measures, we replaced these with the measures of total WMH volumes, total hippocampal volumes (HV), and total brain volumes (BV) in separate multiple regression analyses.

\section{Results}

\section{Baseline sample}

Table 2 shows the coefficient values for each of the predictor variables, when using education as the moderator. Increases in CES-D scores were associated with decreases in language ability and executive function but not memory or visual-spatial ability. Conversely, increases in years of education were associated with increased performance in all of the cognitive domains. There was an interaction between CES-D scores and education for all the cognitive domains except for visual-spatial ability. These interactions revealed that increasing CES-D scores had a greater effect on cognitive performance for individuals with high versus low education (Figures 1 and 3 ).

Table 2 Summary of hierarchical regression analysis for variables predicting cognitive outcomes in the baseline sample $(N=3484)$

\begin{tabular}{|c|c|c|c|c|c|c|c|c|}
\hline \multirow[b]{2}{*}{ Predictor } & \multicolumn{8}{|c|}{ Cognitive outcome } \\
\hline & & $B$ & SE $(B)$ & $\beta$ & Predictor & $B$ & SE $(B)$ & $\beta$ \\
\hline CES-D score & Memory & -0.008 & 0.008 & -0.023 & CES-D score & -0.011 & 0.007 & -0.033 \\
\hline Reading & & 0.013 & 0.001 & $0.301^{\star \star *}$ & Education & 0.038 & 0.004 & $0.263^{\star \star \star}$ \\
\hline $\begin{array}{l}\text { CES-D } \times \text { reading } \\
R^{2}\end{array}$ & & $\begin{array}{c}-0.001 \\
0.21\end{array}$ & 0.000 & $-0.045^{\star}$ & $\begin{array}{l}\text { CES-D } \times \text { education } \\
R^{2}\end{array}$ & $\begin{array}{c}-0.003 \\
0.21\end{array}$ & 0.002 & $-0.044^{*}$ \\
\hline CES-D score & Executive function & -0.022 & 0.010 & $-0.035^{\star}$ & CES-D score & -0.035 & 0.010 & $-0.086^{\star \star \star}$ \\
\hline Reading & & 0.025 & 0.002 & $0.470^{\star \star *}$ & Education & 0.062 & 0.005 & $0.345^{\star \star \star}$ \\
\hline $\begin{array}{l}\text { CES-D } \times \text { reading } \\
R^{2}\end{array}$ & & $\begin{array}{c}-0.002 \\
0.36\end{array}$ & 0.001 & $-0.080^{\star \star \star}$ & $\begin{array}{l}\text { CES-D } \times \text { education } \\
R^{2}\end{array}$ & $\begin{array}{c}-0.001 \\
0.31\end{array}$ & .002 & $-0.067^{\star \star}$ \\
\hline CES-D score & Visual-spatial & 0.000 & 0.006 & -0.001 & CES-D score & -0.005 & 0.006 & -0.018 \\
\hline Reading & & 0.020 & 0.001 & $0.575^{\star \star \star}$ & Education & 0.056 & 0.003 & $0.452^{\star \star \star}$ \\
\hline $\begin{array}{l}\text { CES-D } \times \text { reading } \\
R^{2}\end{array}$ & & $\begin{array}{l}0.000 \\
0.40\end{array}$ & 0.000 & -0.027 & $\begin{array}{l}\text { CES-D } \times \text { education } \\
R^{2}\end{array}$ & $\begin{array}{l}-0.001 \mathrm{E} \\
0.37\end{array}$ & 0.001 & 0.000 \\
\hline CES-D score & Language & -0.007 & 0.006 & -0.022 & CES-D score & -0.015 & 0.006 & $-0.049^{\star \star}$ \\
\hline Reading & & 0.026 & 0.001 & $0.662^{\star \star \star}$ & Education & 0.069 & 0.003 & $0.521^{\star \star \star}$ \\
\hline $\begin{array}{l}\text { CES-D } \times \text { reading } \\
R^{2}\end{array}$ & & $\begin{array}{c}-0.001 \\
0.47\end{array}$ & 0.000 & $-0.066^{\star \star \star}$ & $\begin{array}{l}\text { CES-D } \times \text { education } \\
R^{2}\end{array}$ & $\begin{array}{c}-0.004 \\
0.44\end{array}$ & 0.001 & $-0.065^{\star \star \star}$ \\
\hline
\end{tabular}

Note: All analyses were controlled for age, gender, and race. Reading, education, and CES-D scores were centered at their means. CES-D, Center for Epidemiologic Studies Depression Scale.

${ }^{*} p<0.05 .{ }^{* *} p<0.01 .{ }^{* * *} p<0.001$. 


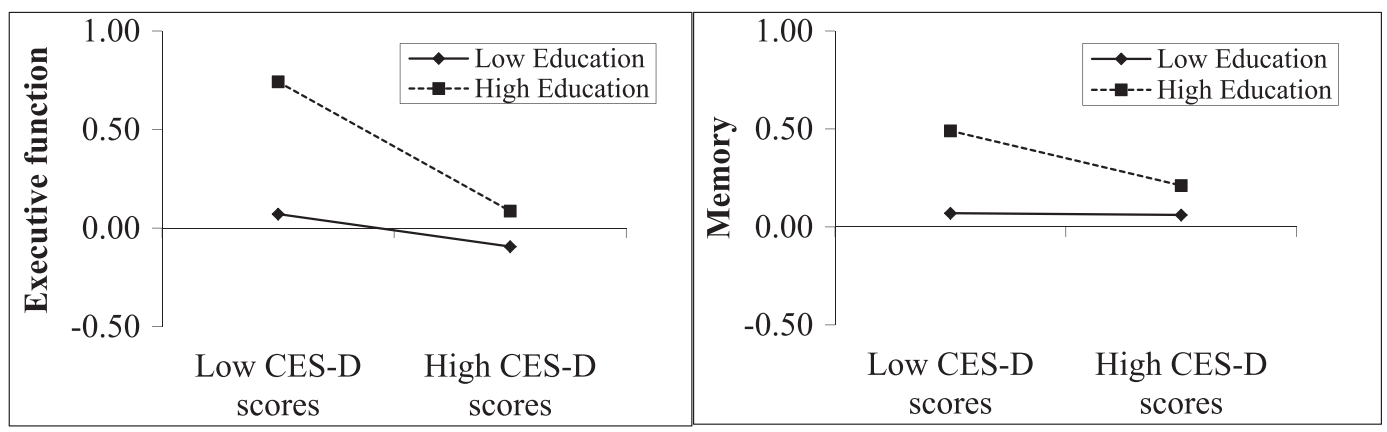

Figure 1 The left panel represents the moderating effect of education on the relationship between depressive symptoms and executive function. The right panel represents the moderating effect of education on the relationship between depressive symptoms and memory. CES-D, Center For Epidemiologic Studies Depression Scale.

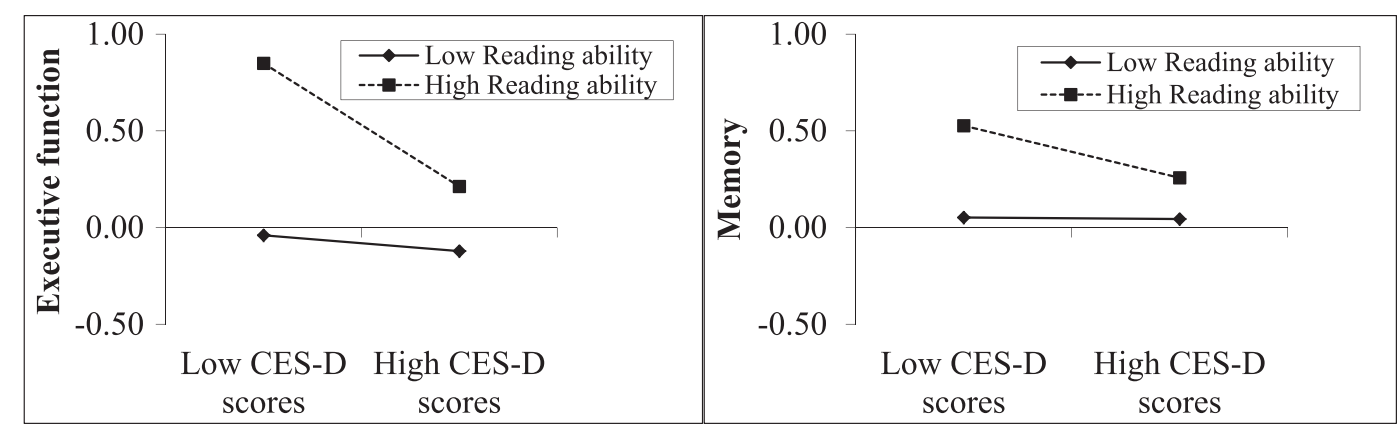

Figure 2 The left panel represents the moderating effect of reading on the relationship between depressive symptoms and executive function. The right panel represents the moderating effect of reading level on the relationship between depressive symptoms and memory performance. CES-D, Center For Epidemiologic Studies Depression Scale. The measure of all cognitive abilities scores was standardized so that negative frequency counts were possible.

Using reading level as the moderator, similar trends were noted in our analyses (Table 2). Increases in CES-D scores were associated with decreases in executive function but not with any of the other cognitive performance measures. Increases in reading scores were associated with better performance on all of the cognitive measures. An interaction effect between
CES-D and reading scores on executive function (Figure 2, left panel), memory (Figure 2, right panel), and language (Figure 3, right panel) but, again, not visual-spatial ability was revealed. Those with greater reading scores showed greater decreases in cognitive performance as depressive symptoms increased.
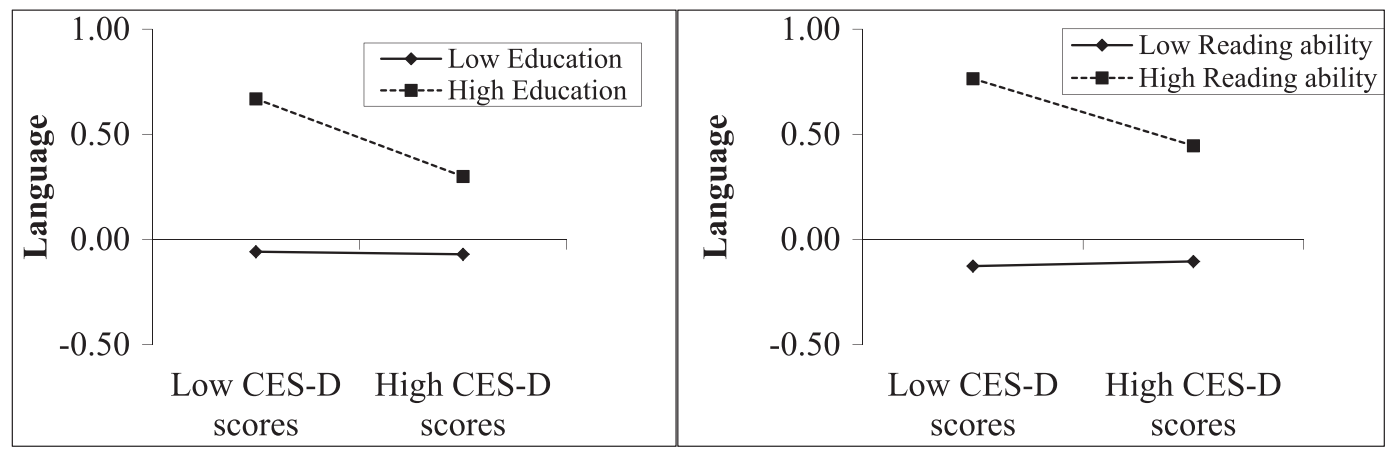

Figure 3 The right panel represents the interaction between years of education and depressive symptoms on language performances. The right panel represents the interaction between reading ability and depressive symptoms on language performance. CES-D, Center For Epidemiologic Studies Depression Scale. The moderator values (i.e., reading and education) are the sample mean plus/minus one standard deviation from the mean. All graphs were generated using MODPROBE, a graphical tool designed for plotting two-way interactions (Hayes and Matthes, 2009). 


\section{Magnetic resonance imaging subsample}

A significant main effect for both education and reading on all four of the cognitive performance measures $(p<0.001)$ in each of the regression analyses was revealed (Table 3 ). CES-D scores were inversely associated with executive function when either education or reading was used in the model but not with any of the other cognitive domains. However, no significant interaction effects between either of the CR measures and CES-D scores were revealed with any of the cognitive outcome measures. However, there was a trend for significance $(p=0.069)$ for the interaction term 'reading by CES-D' on memory performance, in a similar direction to that reported from the baseline data.

Total WMH volumes, HV, and BV were then used as the dependent variables in the analyses. When BV was used as the outcome measure and education was used as the moderator, there was a significant positive association with education $(p=0.003)$. There were no other main effects for either education or CES-D scores on total WMH or total HV. When the reading variable was used as the moderator in the models, there were no significant main effects of CES-D scores on WMH volumes, BV, or HV. However, reading was positively and significantly associated with total HVs and total BVs, $p=0.048$ and $p=0.002$, respectively. There were no statistically significant effects for either of the interaction terms (i.e., education by CES-D and reading by CES-D), on any of the brain variables. However, the interaction between reading and CES-D scores was of borderline significance $(p=0.062)$ when total BV was used as the outcome measure. This observation indicated that those with lower reading scores exhibited a decrease in brain volume as depressive symptoms increased, while this effect was minimized in those with increasing reading scores.

\section{Discussion}

The novelty of this study relates to investigating whether CR moderates the relationship between depressive symptoms and cognition, within a large, racially diverse, community-based sample. An additional novelty relates to investigating whether CR acts as a moderator in the association between depressive symptoms and specific volumetric brain measures. Contrary to what we predicted, we found that those who were more highly educated and/or had greater reading scores showed greater decrements in cognitive performance as depressive symptoms increased than those with lower years of education or reading scores in the baseline sample. While Bhalla et al. (2005) reported that greater education does not buffer the effect of depressive symptoms on cognitive performance, the authors did not report that those with greater education showed more decrements in cognitive performance relative to those with lower years of education. Therefore, these results are not entirely consistent with our finding.

However, findings more closely related to this investigation come from two separate studies that showed that depressive symptoms, within a longitudinal design, were associated with an increased risk of cognitive decline but only in those who were more highly educated (Geerlings et al., 2000a, 2000b).

Table 3 Summary of hierarchical regression analysis for variables predicting brain volumes in the MRI subsample $(N=703)$

\begin{tabular}{|c|c|c|c|c|c|c|c|c|}
\hline \multirow[b]{2}{*}{ Predictor } & \multicolumn{8}{|c|}{ Outcome measure (brain volumes) } \\
\hline & & $B$ & SE $(B)$ & $\beta$ & Predictor & $B$ & SE $(B)$ & $\beta$ \\
\hline CES-D score & Total brain volume & 1.033 & 2.023 & 0.018 & CES-D score & -0.729 & 1.975 & -0.013 \\
\hline Reading & & 12.492 & 3.419 & $0.131^{\star \star *}$ & Education & 2.126 & 0.815 & $0.107^{\star \star}$ \\
\hline $\begin{array}{l}\text { CES-D } \times \text { reading } \\
R^{2}\end{array}$ & & $\begin{array}{l}3.811 \\
0.26\end{array}$ & 2.072 & 0.065 & $\begin{array}{l}\text { CES-D } \times \text { education } \\
R^{2}\end{array}$ & $\begin{array}{c}-0.329 \\
0.25\end{array}$ & 0.405 & -0.028 \\
\hline CES-D score & WMH volume & 0.039 & 0.021 & 0.076 & CES-D score & 0.031 & 0.021 & 0.059 \\
\hline Reading & & -0.041 & 0.036 & -0.047 & Education & 0.009 & 0.008 & 0.052 \\
\hline $\begin{array}{l}\text { CES-D } \times \text { reading } \\
R^{2}\end{array}$ & & $\begin{array}{l}0.000 \\
0.049\end{array}$ & 0.022 & 0.001 & $\begin{array}{l}\text { CES-D } \times \text { education } \\
R^{2}\end{array}$ & $\begin{array}{l}0.002 \\
0.04\end{array}$ & 0.004 & 0.021 \\
\hline CES-D score & Total hippocampal volume & -0.002 & 0.017 & -0.004 & CES-D score & -0.013 & 0.017 & -0.031 \\
\hline Reading & & 0.068 & 0.029 & $0.097^{\star \star}$ & Education & 0.009 & 0.007 & 0.058 \\
\hline $\begin{array}{l}\text { CES-D } \times \text { reading } \\
R^{2}\end{array}$ & & $\begin{array}{r}-0.016 \\
0.062\end{array}$ & 0.017 & -0.036 & $\begin{array}{l}\text { CES-D } \times \text { education } \\
R^{2}\end{array}$ & $\begin{array}{c}-0.005 \\
0.06\end{array}$ & 0.003 & -0.052 \\
\hline
\end{tabular}

Note: All analyses were controlled for age, gender, and race. Reading, education, and CES-D scores were centered at their means. CES-D, Center for Epidemiologic Studies Depression Scale; WMH, white matter hyperintensities; MRI, magnetic resonance imaging.

${ }^{*} p<0.05 .{ }^{* *} p<0.01 .{ }^{* * *} p<0.001$. 
One interpretation of our findings could be that those with greater $\mathrm{CR}$ show more impairment in cognitive performance as symptoms increase because they have greater to 'fall'. The impact of depressive symptoms on those with lower CR may not be as apparent as those with greater CR, as they are already performing at a lower cognitive level. We may also speculate that low mood impaired cognitive performance, that is, disruptions in more fundamental processes such as attention and/or processing speed due to low mood or distracting thoughts rather than neuropsychological impairment per se (Nebes et al., 2000). Again, these disruptions may be more pertinent to those performing at a higher cognitive level. One final explanation may be that those with greater CR may be more likely to experience depression as cognitive impairment increases.

While there was some discrepancy between the findings in baseline and subsample (i.e., no significant interactions were revealed in the subsample), this may be accounted for by a lack of power. Equally, it could have also been due to an overall significantly lower mean CES-D score in this subsample compared with the baseline sample. Perhaps a larger sample or higher mean CES-D score would better reveal this association and yield some clarity to this particular issue.

The marginally significant finding that those with lower reading scores showed a greater decrease in total brain volume than those with greater reading ability may be of interest. Much research has shown that both regional (Janssen et al., 2007) and overall structural changes (Pantel et al., 1997) are related to the onset of some 'subtypes' of depression (Krishnan, 2002; Alexopoulos et al., 2004; Geerlings et al., 2012). Perhaps, lower CR individuals may be more at risk of this type of 'biological'-related depression. However, the direction of this association is far from clear. It may be that depression results in volume loss, rather than the reverse. Nevertheless, understanding this subtype of depression in the context of CR may aid our understanding of the mechanisms that contribute to some causes of depression and not others. The findings from the present study provide some tentative justification for exploring this further.

Similarities and differences in results derived when using education versus reading performance

We employed two common proxies for CR in the present study-reading ability and education. The findings were almost identical when either CR proxy was used in our analyses. For example, there was a significant interaction effect between depressive symptoms and both of the CR variables for memory, executive function, and language performance but not visual-spatial ability in the baseline sample. These similarities are further illustrated by the shape of the regression slopes (as depicted in Figures 1, 2 and 3) when either reading or education was used as the moderating variable. While no significant results for these analyses were revealed in the subsample, there was a borderline significant finding for the interaction between reading and CES-D on both memory performance and total BV. However, these two trends were not evident when education was used as the moderating variable, perhaps indicating that reading ability may be a more sensitive proxy for CR in the present sample. This is in line with previous research that showed in a subsample of the WHICAP study that reading level was a better indicator of decline in memory, executive function, and language performance than years of education (Manly et al., 2005). Nevertheless, this difference between the CR variables was less apparent in the cross-sectional design of the present study. However, future research aimed at exploring this topic from a longitudinal perspective may benefit from a closer consideration of the chosen $\mathrm{CR}$ variable.

\section{Limitations}

The cross-sectional design of this study is an obvious limitation insofar as elucidating the pathways, both cognitive and biological, in which depressive symptoms influence cognition as a function of CR. In addition, the findings from participants reporting depressive symptoms may not be generalizable to those with clinical depression; it may be that greater severity of depressive symptoms would result in a different cognitive outcome for the individual irrespective of their level of CR. However, investigating the role of CR in clinical depression on both cognition and neuroanatomical structures would be worthwhile for future research. A further consideration in interpreting the results of the study is that despite the broad range of education level in the present sample, the mean years of education was relatively low. This is explained by the majority of the sample being non-White, categorized as either African American or Hispanic composing approximately $72 \%$ of the sample. These groups together have a lower mean education ( 7 years) than the White group (13 years). It is possible that there may be some culturally based differences related to educational experience that may have impacted neuropsychological test performances. Future research could examine how the interaction between depression and CR on neuropsychological performance may vary within these ethnic groups. 


\section{Conclusion}

Our findings indicate that the association between late life depressive symptoms and core aspects of cognition varies depending on one's level of CR. Those that had greater levels of education and/or reading ability showed a greater decrease in memory, executive function, and language performances as depressive symptoms increased than those with lower years of education and reading ability.

\section{Conflict of interest}

None declared.

\section{Key points}

- Cognitive reserve influenced the association between depressive symptoms and core aspects of cognition, but this association was the inverse of what we had predicted.

- Those with greater levels of cognitive reserve showed decrements in cognitive performances as depressive symptoms increased, while this effect was minimal in those with lower levels of cognitive reserve.

- While there were no significant interaction effects between the measures of cognitive reserve and depressive symptoms, a borderline interaction effect between reading level and depressive symptoms on total brain volume indicated that those with lower reading levels showed a decrease in brain volume as depressive symptoms increased.

- Future research may clarify the potential association between late-life depressive symptoms and brain volumes in the context of cognitive reserve in a larger sample and/or with participants endorsing a higher mean depressive symptom score than in the present study sample.

\section{References}

Alexopoulos GS, Kiosses DN, Murphy C, Shanmugham B, Heo M. 2004. Executive dysfunction, heart disease burden, and remission of geriatric depression. Neuropsychopharmacology 29: 2278-2284.

Becker JT, Chang YF, Lopez OL, et al. 2009. Depressed mood is not a risk factor for incident dementia in a community-based cohort. Am J Geriatr Psychiatry: Off J Am Assoc Geriatr Psychiatry 8: 653.

Bhalla RK, Butters MA, Zmuda MD, et al. 2005. Does education moderate neuropsychological impairment in late-life depression?. Int J Geriatr Psychiatry 5: 413-417.

Bieliauskas LA, Lamberty G, Boczar J. 1991. Lack of depression effects on cognitive functions in the elderly (Abstract). J Clin Exp Neuropsychol 13: 433.
Biringer E, Mykletun A, Dahl AA, et al. 2005. The association between depression, anxiety, and cognitive function in the elderly general population-the Hordaland Health Study. Int J Geriatr Psychiatry 10: 989-997.

Boone KB, Lesser IM, Miller BL, et al. 1995. Cognitive functioning in older depressed outpatients: relationship of presence and severity of depression to neuropsychological test scores. Neuropsychology 3: 390.

Brickman AM, Schupf N, Manly JJ, et al. 2008. Brain morphology in older African Americans, Caribbean Hispanics, and whites from northern Manhattan. Arch Neurol 8: 1053.

Butters MA, Whyte EM, Nebes RD, et al. 2004. The nature and determinants of neuropsychological functioning in late-life depression. Arch Gen Psychiatry 6: 587.

DeCarli C, Maisog J, Murphy DG, et al. 1992. Method for quantification of brain, ventricular, and subarachnoid CSF volumes from MR images. I Comput Assist Tomogr 2: 274-284.

DeCarli C, Murphy DG, Teichberg D, Campbell G, Sobering GS. 1996. Local histogram correction of MRI spatially dependent image pixel intensity nonuniformity. J Magn Reson Imaging 6: 519-528.

DeCarli DG, Murphy M, Tranh CL et al. 1995. The effect of white matter hyperintensity volume on brain structure, cognitive performance, and cerebral metabolism of glucose in 51 healthy adults. Neurology 11: 2077-2084.

Del Ser T, González-Montalvo JI, Martínez-Espinosa S, Delgado-Villapalos C, Bermejo F. 1997. Estimation of premorbid intelligence in Spanish people with the Word Accentuation Test and its application to the diagnosis of dementia. Brain Cogn 3: 343-356.

Dufouil C, Fuhrer R, Dartigues JF, Alpérovitch A. 1996. Longitudinal analysis of the association between depressive symptomatology and cognitive deterioration. Am J Epidemiol 7: 634-641.

Ganguli M, Du Y, Dodge HH, Ratcliff GG, Chang CC. 2006. Depressive symptoms and cognitive decline in late life: a prospective epidemiological study. Arch Gen Psychiatry 63: 153-160.

Geerlings MI, Bouter LM, Schoevers R, et al. 2000a. Depression and risk of cognitive decline and Alzheimer's disease Results of two prospective community-based studies in the Netherlands. Br J Psychiatry 6: 568-575.

Geerlings MI, Brickman AM, Schupf N, et al. 2012. Depressive symptoms, antidepressant use, and brain volumes on MRI in a population-based cohort of old persons without dementia. J Alzheimers Dis 1: 75-82.

Geerlings MI, Schmand B, Braam AW, et al. 2000b. Depressive symptoms and risk of Alzheimer's disease in more highly educated older people. J Am Geriatr Soc 9: 1092-1097.

Geffen G, Bate A, Wright M, Rozenbilds U, Geffen L. 1993. A comparison of cognitive impairments in dementia of the Alzheimer type and depression in the elderly. Dementia 4: 294-300.

Green RC, Cupples LA, Kurz A, et al. 2003. Depression as a risk factor for Alzheimer disease: the MIRAGE Study. Arch Neurol 5: 753.

Hart RP, Kwentus JA, Hamer RM, Taylor J. 1987. Selective reminding procedure in depression and dementia. Psychol Aging 2: 111-115.

Hayes AF, Matthes J. 2009. Computational procedures for probing interactions in OLS and logistic regression: SPSS and SAS implementations. Behavior Research Methods 41: 924-936.

Herrmann LL, Le Masurier M, Ebmeier KP. 2008. White matter hyperintensities in late life depression: a systematic review. J Neurol Neurosurg Psychiatry 6: 619-624.

Irwin M, Artin KH, Oxman MN. 1999. Screening for depression in the older adult: criterion validity of the 10-item Center for Epidemiological Studies Depression Scale (CES-D). Arch Intern Med 15: 1701.

Janssen J, Pol HEH, de Leeuw FE, et al. 2007. Hippocampal volume and subcortical white matter lesions in late life depression: comparison of early and late onset depression. J Neurol Neurosurg Psychiatry 6: 638-640.

Jansson M, Gatz M, Berg S, et al. 2003. Association between depressed mood in the elderly and a 5-HTR2A gene variant. Am J Med Genet B Neuropsychiatr Genet 11: 79-84.

Kessler RC, Foster CL, Saunders WB, Stang PE. 1995. Social consequences of psychiatric disorders, I: educational attainment. Am J Psychiatry 7: 1026-1032.

Kim JM, Shin IS, Yoon JS, Stewart R. 2002. Prevalence and correlates of late-life depression compared between urban and rural populations in Korea. Int J Geriatr Psychiatry 5: 409-415.

Krishnan KRR. 2002. Biological risk factors in late life depression. Biol Psychiatry 3: $185-192$.

Ladin K. 2008. Risk of late-life depression across 10 European Union countries: deconstructing the education effect. J Aging Health 6: 653-670.

Lee JS, Potter GG, Wagner HR, Welsh-Bohmer, KA, Steffens DC. 2007. Persistent mild cognitive impairment in geriatric depression. Int Psychogeriatr 19: 125-135.

Lindsay J, Laurin D, Verreault R, et al. 2002. Risk factors for Alzheimer's disease: a prospective analysis from the Canadian Study of Health and Aging. Am J Epidemiol 5: $445-453$.

Lloyd AJ, Ferrier IN, Barber R, et al. 2004. Hippocampal volume change in depression: late-and early-onset illness compared. Br J Psychiatry 6: 488-495.

Lockwood KA, Alexopoulos GS, Kakuma T, Van Gorp WG. 2000. Subtypes of cognitive impairment in depressed older adults. Am J Geriatr Psychiatry 3: 201-208. 
Manly JJ, Schupf N, Tang MX, Stern Y. 2005. Cognitive decline and literacy among ethnically diverse elders. J Geriatr Psychiatry Neurol 4: 213-217.

Nebes RD, Butters MA, Mulsant BH, et al. 2000. Decreased working memory and processing speed mediate cognitive impairment in geriatric depression. Psychol Med 3: 679-691.

Ownby RL, Crocco E, Acevedo A, John V, Loewenstein D. 2006. Depression and risk for Alzheimer disease: systematic review, meta-analysis, and metaregression analysis. Arch Gen Psychiatry 5: 530.

Pantel J, Schröder J, Essig M, et al. 1997. Quantitative magnetic resonance imaging in geriatric depression and primary degenerative dementia. J Affect Disord 1: 69-83. Pinquart M, Sörensen S. 2000. Influences of socioeconomic status, social network, and competence on subjective well-being in later life: a meta-analysis. Psychol Aging 2: 187.

Raskin A. 1986. Partialing out the effects of depression and age on cognitive functions: experimental data and methodologic issues. Handbook for Clinical Memory Assessment Of Older Adults, Poon LW, Crook T, Davis KL, Eisdorfer C, Gurland BJ, Kaszniak AW, Thompson LW (ed.). American Psychological Association, 419: Washington, DC, US; pp. 244-256.

Sheline YI, Barch DM, Garcia K, et al. 2006. Cognitive function in late life depression: relationships to depression severity, cerebrovascular risk factors and processing speed. Biol Psychiatry 1: 58-65.

Stern Y. 2003. The concept of cognitive reserve: a catalyst for research. J Clin Exp Neuropsychol 5: 589-593.
Stern Y, Andrews H, Pittman J, et al. 1992. Diagnosis of dementia in a heterogeneous population: development of a neuropsychological paradigm-based diagnosis of dementia and quantified correction for the effects of education. Arch Neurol 5: 453.

Tang MX, Cross P, Andrews H, et al. 2001. Incidence of AD in African-Americans, Caribbean Hispanics, and Caucasians in northern Manhattan. Neurology 1: 49-56.

Thompson C, Syddall H, Rodin IAN, Osmond C, Barker DJ. 2001. Birth weight and the risk of depressive disorder in late life. Br J Psychiatry 5: 450-455.

Turner RJ, Lloyd DA. 1999. The stress process and the social distribution of depression. J Health Soc Behav 40: 374-404.

Wight RG, Aneshensel CS, Seeman TE. 2002. Educational attainment, continued learning experience, and cognitive function among older men. J Aging Health 2: 211-236.

Wilkinson GS. 1993. The Wide Range Achievement Test (WRAT-3): administration manual, Wilmington, DE, Wide Range.

\section{Supporting information}

Additional supporting information may be found on the online version of this article at the publisher's web site. 